
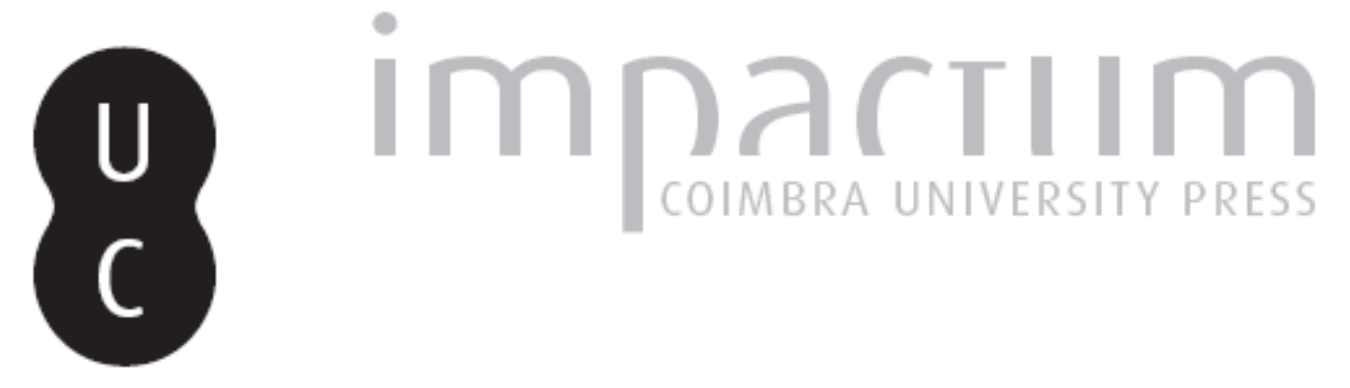

De l'union des îles à leur séparation: l'organisation administrative de la Corse et de la Sardaigne au ler siècle

\author{
Autor(es): $\quad$ Michel, François
}

Publicado por: Faculdade de Letras da Universidade de Coimbra

URL persistente:

URI:http://hdl.handle.net/10316.2/37865

DOI:

DOI:http://dx.doi.org/10.14195/1647-8657_49_9

Accessed : $\quad$ 26-Apr-2023 13:20:46

A navegação consulta e descarregamento dos títulos inseridos nas Bibliotecas Digitais UC Digitalis, UC Pombalina e UC Impactum, pressupõem a aceitação plena e sem reservas dos Termos e Condições de Uso destas Bibliotecas Digitais, disponíveis em https://digitalis.uc.pt/pt-pt/termos.

Conforme exposto nos referidos Termos e Condições de Uso, o descarregamento de títulos de acesso restrito requer uma licença válida de autorização devendo o utilizador aceder ao(s) documento(s) a partir de um endereço de IP da instituição detentora da supramencionada licença.

Ao utilizador é apenas permitido o descarregamento para uso pessoal, pelo que o emprego do(s) título(s) descarregado(s) para outro fim, designadamente comercial, carece de autorização do respetivo autor ou editor da obra.

Na medida em que todas as obras da UC Digitalis se encontram protegidas pelo Código do Direito de Autor e Direitos Conexos e demais legislação aplicável, toda a cópia, parcial ou total, deste documento, nos casos em que é legalmente admitida, deverá conter ou fazer-se acompanhar por este aviso.

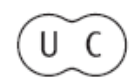


CONIMBRIGA

C)

CN N N

INSTITUTO DE ARQUEOLOGIA

VOLUME XLIX • 2010

FACULDADE DE LETRAS 
FRANÇOIS MiCHEL

DE L'UNION DES ÎLES À LEUR SÉPARATION.

L'ORGANISATION ADMINISTRATIVE

DE LA CORSE ET DE LA SARDAIGNE AU I Ir SIÈCLE

"Conimbriga" XLIX (2010) p. 161-181

RÉSUMÉ: Les îles de Corse et de Sardaigne, après avoir été gouvernées dans un cadre provincial unique, ont vu leurs administrations se séparer au cours $\mathrm{du} \mathrm{I}^{\mathrm{er}}$ siècle. Il est encore malaisé de déterminer avec précision à quelle date a eu lieu ce remaniement administratif, et quels événements ont poussé le gouvernement impérial à l'effectuer. Cette étude principalement fondée sur le dossier épigraphique a tout d'abord pour objet d'identifier quelles étapes ont entériné la division administrative des deux îles, placées sous l'autorité successive d'un proconsul, d'un prolégat, de préfets aux attributions diverses, enfin de procurateurs, et quels événements ont pu se trouver à l'origine de si nombreuses mutations, que l'on qualifierait plus volontiers d'adaptations. Un éclairage inédit vient de l'étude des carrières et des titres des gouverneurs ainsi que de l'analyse de la hiérarchie existant entre les divers commandements mentionnés par les textes, et nous permet d'envisager une datation plus précise de la réforme qui a abouti à la mise en place d'un nouvel ordre provincial.

Resumo: As ilhas da Córsega e da Sardenha, depois de terem sido governadas num quadro provincial único, viram no decurso do século I as suas administrações serem separadas. Não é fácil definir em que data precisa ocorreu este rearranjo administrativo nem determinar os acontecimentos que levaram o governo imperial a determiná-lo. Este estudo, fundamentalmente baseado na informação epigráfica, propõe-se identificar as etapas de homologação da divisão administrativa das duas ilhas, colocadas sob a autoridade sucessiva de um procônsul, de um prolegado, de prefeitos com atribuições diversas e de procuradores e, quais os acontecimentos que se podem encontrar na origem de numerosas mutações a que se ajusta melhor o qualificativo de adaptações. O estudo

Conimbriga, 49 (2010) 161-181 
das carreira e dos títulos dos governadores, bem como a análise da hierarquia existente entre os diversos comandos mencionados pelos textos, traz-nos uma visão inédita que nos permite apontar uma datação mais precisa da reforma que se traduziu pela instalação de uma nova ordem provincial. 


\title{
DE L'UNION DES ÎLES À LEUR SÉPARATION L'ORGANISATION ADMINISTRATIVE DE LA CORSE ET DE LA SARDAIGNE AU Ier SIÈCLE
}

\author{
À u mo anzianu professore é amicacciu Robert Étienne \\ in téstimoniu di a mo fideltà.
}

Territoire conquis sur Carthage, jumelé avec la Sardaigne, administré par des préteurs ou des propréteurs, sinon par des consuls, la Corse a toujours sous la République eu des gouverneurs issus du sénat ${ }^{1}$. Auguste, en 27 av. J.-C., a maintenu cette administration sénatoriale en confiant la province à des proconsuls. Mais ceux-ci cèdent la place en l'année 6 de notre ère à des membres de l'ordre équestre censés mieux contrôler militairement les îles et éradiquer le brigandage. C'est lors de l'élaboration du corpus épigraphique des îles de Corse ${ }^{2}$ et de Sardaigne que nous avons eu l'occasion de constater de notables différences dans l'énoncé des titulatures de ces gouverneurs équestres. Il n'y avait alors qu'un pas à franchir pour supposer que la modification

1 La liste de ces gouverneurs de Corse et Sardaigne se trouve dans l'ouvrage de T.R.S. Broughton, The magistrates of the Roman Republic, I-III, Supplements, Atlanta, 1952-1986, et un proconsul est à ajouter entre 121 et 116 av. J.-C.: M. Cornu[ficius. f.] / pro $c[o(n) s(u l e)]$ figure sur une borne milliaire de Cuglieri (Oratiddo), cf. A. Mastino, Storia della Sardegna antica, Nuoro, 2005, p. 157 et 377-378, puis A. Corda, A. Mastino, Il più antico miliario dalla Sardegna dalla strada a Tibulas Sulcos, dans Contributi all'epigrafia d'età Augustea, Actes de la XIIIe rencontre franco-italienne sur l'épigraphie du monde romain (Macerata, 9-11 septembre 2005), Tivoli, 2007, p. 277-314 = AE 2007, 693 .

2 Ce corpus a fait l'objet d'une thèse de Doctorat sur le sujet «Les inscriptions latines et grecques de la Corse romaine» soutenue le 17 décembre 2011 et en cours de publication.

Conimbriga, 49 (2010) 161-181 
de ces titulatures se soit effectuée selon des données chronologiques, et c'est tout naturellement que nous nous sommes interrogé sur la nature de l'administration des îles et sur son évolution. Faute d'avoir trouvé dans les multiples parutions consultées une solution satisfaisante, nous avons nous-même tenté de répondre à cette question et avons donc dû mener un débat à plusieurs entrées. Il s'est tout d'abord agi de déterminer si, au I ${ }^{\text {er }}$ siècle, l'administration de la Corse a été séparée de celle de la Sardaigne. Cette question, dont la réponse se présente de manière évidente en 67 , lorsque la Sardaigne retourne sous l'autorité du Sénat alors que la Corse reste sous administration impériale directe, reste non résolue pour les cinquante années qui précèdent. Cependant, certaines titulatures de gouverneurs antérieures à cette date donnent des indications très précises quant aux compétences géographiques de ceux-ci, et ce fait tend à nous faire supposer que les deux îles connaissent des gouvernements différents antérieurement à l'année 67. La question s'articule alors autour d'une autre interrogation: si cette séparation a eu lieu précédemment, quand donc s'est-elle effectuée, et quels sont les éléments qui nous permettraient d'en déterminer le moment? Nous verrons que ces derniers, pour subtils qu'ils soient, n'en présentent pas moins un caractère bien suggestif. Enfin, pour rendre la chose plus directement admissible, il restait à déterminer pour quels motifs et dans quelles conditions cette séparation s'est effectuée. Faire le point sur ce dernier aspect nous permettait en effet de donner une solution admissible à tout point de vue.

Le point le plus important reste cependant le suivant: au premier siècle, l'histoire de la Corse ne peut pas être traitée indépendamment de celle de la Sardaigne, et la réciproque est également vraie ${ }^{3}$. Outre l'éclairage sur la politique impériale telle qu'elle a été menée envers les deux îles et les conclusions que l'on pourra en tirer, l'intérêt principal de cette étude est d'être la première à prendre en compte les deux entités comme base de raisonnement.

Ainsi pourrons-nous proposer d'éventuelles solutions en fonction des lectures tirées des corpus épigraphiques, en premier lieu par l'étude de l'évolution des modes de gouvernement tels que nous la présentent les sources et la bibliographie récente puis, dans un second temps,

3 A. Mastino, dans R. Zucca, La Corsica romana, Oristano, 1996, p. 7 [ = Corsica romana $]$ évoque fugitivement ce thème sans que son contenu soit réellement pris en compte dans l'ouvrage.

Conimbriga, 49 (2010) 161-181 
en considérant le contexte politique méditerranéen durant les deux premiers tiers du I ${ }^{\text {er }}$ siècle, où nous trouverons peut-être les motifs de ces changements.

\section{Rome et la province de Corse et Sardaigne: le débat}

C'est à l'issue de la première guerre punique que Rome met la main sur la Sardaigne et la Corse dans des circonstances jugées dès l'Antiquité peu honorables ${ }^{4}$. Ce n'est qu'à la suite de plusieurs expéditions militaires, alors que les conquêtes sont entérinées, que les deux îles sont constituées en une seule province ${ }^{5}$. Les opérations de pacification dureront cependant jusqu'au règne de Tibère.

En 27 av. J.-C., la province de Corse et Sardaigne est une province bicéphale confiée au Sénat qui en assure l'administration par le biais d'un proconsul de rang prétorien dont le siège se trouve à Karales, capitale de la province. Nous connaissons pour cette période d'administration sénatoriale trois gouverneurs, Q. Caecilius Metellus Creticus, mentionné sur un texte de Karales ${ }^{6}$, C. Mucius Scaevola connu par deux textes, l'un de Nora, l'autre d'Aleria ${ }^{7}$, et [---]rius $\mathrm{Ca}[---]$ dont le nom est relevé à

4 M. Dubuisson, Procédés de la diplomatie romaine: l'annexion de la Sardaigne et le sens de $\sigma v \gamma \kappa \alpha \tau \alpha \beta \alpha i v \varepsilon \imath v$ (Polybe, III, 10, 1), dans REL 1979, p. 123-125 suggère qu'à la faveur de contentieux mineurs, et brandissant la menace de la guerre, les Romains ont profité des difficultés de Carthage pour extorquer des concessions plus importantes que celles que les Puniques, très désireux de faire la paix, s'étaient imaginées; imprudemment, ils s'étaient à l'avance engagés à satisfaire les revendications romaines et, malgré leur dépit, ne pouvaient revenir sur la parole donnée.

5 La province a-t-elle été fondée en 238 ou en 227? Solin (Collectanea rerum memorabilium, V,1, éd. Th. Mommsen, Berlin, 1895, 1958², p. 47-48) écrit que c'est en 227 que l'on met en place l'administration de la Corse et Sardaigne: "Primo quod utraque insula (Sardinia et Sicilia) in Romanorum arbitratum redacta, iisdem temporibus facta provincia est, cum eodem anno Sardiniam M. Valerius, alteram C. Flaminius praetor sortiti sunt". Cf. T. Corey Brennan. The Praetorship in the Roman Republic, New York, 2000.

6 CIL X 7581 (Karales) rappelle un acte d'évergétisme de ce gouverneur.

7 C'est une munificence du même ordre de la part de C. Mucius Scaevola que mentionne CIL X 7543 (Nora) complété par R. Zucca, Additamenta epigraphica all'amministrazione della Sardegna da Augusto all'invasione vandalica, dans Varia Epigraphica, Atti del Colloquio Internazionale di Epigrafia (Bertinoro, 8-10 giugno 2000), éd. G. Angeli Bertinelli et A. Donati, Faïence, 2001, p. 513-535 [=Additamenta

Conimbriga, 49 (2010) 161-181 
Fordongianus ${ }^{8}$. Le contrôle par le Sénat des deux îles a cependant très tôt dû se révéler insuffisant, puisque Auguste, 33 ans après le partage, décide d'une modification du statut de la province.

En effet, en 6 ap. J.-C., selon Dion Cassius 9 , la province de Corse et Sardaigne change de condition juridique. Auguste, désireux de lutter contre la piraterie qui rend ces territoires peu sûrs, reprend les îles sous sa juridiction directe et choisit d'y envoyer un gouverneur extraordinaire que Dion Cassius désigne du nom de $\sigma \tau \rho \alpha \tau \imath \dot{\alpha} \rho \chi \eta \varsigma$. Ce terme, sur lequel nous reviendrons, trouve un parallèle épigraphique latin en 13/14, à la fin du règne d'Auguste: sur un milliaire de Fordongianus, T. Pompeius Proculus est en effet nanti du titre de prolégat ${ }^{10}$.

Puis apparaissent dans les inscriptions une série de préfets en charge soit de la province, sans autre précision ${ }^{11}$, soit de la Corse $^{12}$, soit de la Sardaigne ${ }^{13}$, soit encore d'un district militaire de la Barbaria $^{14}$ ou de la cité de Valentia ${ }^{15}$, sans que l'on ait jusque-là réellement distingué leur hiérarchie et les implications de celle-ci sur l'administration des îles avant que la Sardaigne ne revienne au Sénat.

epigraphica $]=A E, 2001,1110$. L'inscription d'Aleria $(A E 1991,919)$ faisait probablement partie de la décoration du forum et est identifiée par R. Zucca, Corsica romana, p. $232-233, \mathrm{n}^{\circ} 19$.

8 G. Sotgiu, Il clero in Sardegna nelle iscrizioni paleocristiane: un nuovo vescovo da Forum Traiani e nuove acquisizioni epigrafiche, dans La Sardegna paleocristiana tra Eusebio e Gregorio Magno, éd. A. Mastino, G. Sotgiu, N. Spaccapelo, Cagliari, 1999, p. 466 n. $4=A E$ 1999, 804a, et R. Zucca, Additamenta epigraphica, p. 527.

9 Dion Cassius, Histoire romaine, LV, 28, 1, éd. E. Cary, Loeb, Londres, 1960, p. 467.

10 Selon S. Demougin (L'ordre équestre sous les julio-claudiens, Rome, 1988, p. 724, n. 141 [= Ordre équestre]), ce dernier est sous l'autorité du proconsul ou du légat dont dépendent normalement les troupes mises à sa disposition; contra, L. Loreto, Il comando militare nelle province procuratorie (30 a.C. - 280 d.C.). Dimensione militare e dimensione costituzionale, Naples, 2000.

${ }^{11} A E$ 1921, $86=A E$ 1971, 118; $A E$ 1972, 225.

12 CIL XII $2455=$ ILN Vienne, 707 avec la bibliographie complémentaire.

$13 E E$ VIII, 744, $A E$ 1893, 47, et R. Zucca, Due nuovi miliari di Claudio e la data della costruzione della via a Karalis in Sardinia, dans Epigraphica, 64, 2002, p. $57-68=A E 2002,629$ et 630 .

14 CIL XIV, $2954=$ ILS 2684

15 A. Forci et R. Zucca, M. Arrecinus Helius praefectus civitat(is) [Va]le[n]tinae, dans Epigraphica 69 (2007), p. 209-239 = AE 2007, 692.

Conimbriga, 49 (2010) 161-181 
En 67, Néron proclame la liberté des cités grecques et supprime de fait la province d'Achaïe. À titre de compensation, la Sardaigne est remise à l'administration sénatoriale ${ }^{16}$. Ce n'est qu'à ce moment que la division administrative des îles en deux provinces distinctes est rendue parfaitement nette, car c'est alors par statut que les deux îles sont alors séparées. En 69, Tacite mentionne du reste que le procurateur de Corse Decimus Picarius, désireux de rallier la province au parti de Vitellius, cherche à éliminer les opposants à ce projet: la Corse est donc alors une province procuratorienne (Histoires, II, 16). De la même année date l'arbitrage du proconsul de Sardaigne L. Helvius Agrippa connu sous le nom de table d'Esterzili, qui consacre d'une manière claire le fait que l'île soit une province sénatoriale.

Le débat se présente donc d'une manière qui apparaît très simple: au début du I $\mathrm{I}^{\mathrm{er}}$ siècle, la Corse et la Sardaigne forment une unique province; à la fin du deuxième tiers du ${ }^{\text {er }}$ siècle, ces îles sont administrativement séparées. Comment cette évolution s'est-elle effectuée, a-t-elle connu plusieurs étapes, et lesquelles? Une rapide étude bibliographique ne permet pas de présenter un état des lieux satisfaisant.

E. Michon, en 1888, suggère que les deux îles, dès l'an 6 de notre ère, ne forment qu'une seule province procuratorienne jusqu'en 67, lorsque Néron confie la Sardaigne au Sénat ${ }^{17}$. P. Meloni, dès 1953, réalise le premier travail fouillé sur la question de l'administration de la Sardaigne et lie la séparation des deux îles à l'envoi, en 19, de quatre mille affranchis en Sardaigne pour combattre le brigandage. Il estime en outre que l'inscription consacrée au préfet de Corse L. Vibrius Punicus est datable des premiers temps de l'empire, et qu'elle doit être considérée parallèlement à celle qui mentionne le préfet des cités de la Barbaria, Sex. Iulius Rufus ${ }^{18}$. G.E.F. Chilver, en 1979, suggère que la

16 Pausanias, Description de la Grèce, 7.17.3, éd. M. Cazevitz, CUF, Paris, 2000, p. 57; comme seule la Sardaigne a été remise au Sénat, on pourrait voir dans ce fait un argument supplémentaire pour admettre que les deux îles étaient déjà séparées.

17 E. Michon, L'administration de la Corse sous la domination romaine, dans MEFRA VIII (1888), p. 416, mentionne également l'opinion inverse de A. W. Zumpt, Commentationum epigraphicarum ad antiquitates romanas pertinentium volumen, Berlin, 1850/1854, p. 268 selon laquelle les îles ont constitué deux provinces séparées et la juge «sans motifs suffisants».

18 P. Meloni, L'amministrazione della Sardegna nel I sec. d.C., in $A F M L C, \mathrm{XXI}$, 1953, p. 117-147, et plus particulièrement p. 121-123. Il date par ailleurs la dédicace des cités de la Barbaria d'entre 20 et 25.

Conimbriga, 49 (2010) 161-181 
Corse est détachée de la Sardaigne dès l'an 6 de notre ère, et se fonde sur le fait que L. Vibrius Punicus, dont il date l'inscription des débuts de l'époque julio-claudienne, semble être un officier indépendant ${ }^{19}$. C. Vismara, en 1987, avec le même argument, pense que la Corse a eu une administration propre avant 67; elle ne propose pas de date précise, mais fait le lien avec L. Aurelius Patroclus, préfet de Sardaigne en $46^{20}$. D.B. Saddington évoque les données du problème sans donner de réponse satisfaisante. Il se borne en effet à conclure qu' «au début du principat, il n'est pas bon de chercher trop d'uniformité» ${ }^{21}$. R. Zucca, plus récemment, pense que c'est dès 6 ap. J.-C. qu'Auguste a subdivisé la province sénatoriale en deux provinces procuratoriennes ${ }^{22}$. Encore plus récemment, A. Mastino écrit que la province est peut-être autonome dès le règne d'Auguste, mais que d'autres auteurs pensent que c'est à partir du règne de Tibère; il définit la dédicace des cités de la Barbaria comme un acte d'hommage au prince après les succès remportés par celui-ci, mais préfère penser qu'elle date de l'époque d'Auguste et suggère finalement que le prolégat existe encore en 14 ap. J.-C. ${ }^{23}$.

Les nombreux auteurs qui ont abordé cette question ont donc manifesté des opinions très différentes et l'unanimité s'est encore moins faite lorsque certains d'entre eux sont revenus sur leurs positions.

\section{Les titres des gouverneurs et leur ressort territorial}

À partir du moment où les îles ne sont plus gouvernées par des proconsuls, leur administration se militarise sous la surveillance directe du pouvoir impérial. En 6 de notre ère, Auguste les confie à des $\sigma \tau \rho \alpha \tau \imath \alpha ̉ \rho \chi \varepsilon \imath \varsigma$ que Dion Cassius définit comme originaires de l'ordre

19 G.E.F. Chilver, A historical commentary on Tacitus' Histories I and II, Oxford, 1979, p. 181.

20 C. Vismara, Funzionari civili e militari nella Corsica romana, dans Bollettino di Numismatica, Studi per Laura Breglia, vol. III, 1987, p. 62 n. 32.

21 D.B. Saddington, The armed forces and the equestrian administrators of early imperial Sardinia and Corsica, dans Sardinia antiqua, Cagliari, 1992, p. 265-270.

22 R. Zucca, Corsica romana, p. 116. Il fait référence à P. Meloni, La Sardegna romana, Sassari, 1991, p. 142.

23 A. Mastino, Storia della Sardegna antica, Nuoro, 2005, p. 126.

Conimbriga, 49 (2010) 161-181 
équestre ${ }^{24}$. Ce terme, que Dion Cassius n'utilise pas fréquemment, et pratiquement toujours dans un contexte de conflit aigu, désigne des subordonnés équestres aux ordres directs de l'empereur ou du général en chef, des chevaliers pourvus de pouvoirs militaires, sans avoir pourtant l'imperium d'un propréteur ou d'un proconsul, qui font fonction de légat et ont pu commander des légionnaires comme des auxiliaires ${ }^{25}$. La place de ces $\sigma \tau \rho \alpha \tau \iota \dot{\alpha} \rho \chi \varepsilon \iota \varsigma$ dans la hiérarchie des commandements militaires peut être identifiée à l'aide d'une inscription, un milliaire retrouvé à Fordongianus, au départ réputée fausse, puis authentifiée (CIL X, 1451*). Elle mentionne un officier portant le titre de prolégat, T. Pompeius Proculus, et est bien datée entre le 26 juin 13 et le 25 juin 14. Le prolégat, étudié par ailleurs, est un gouverneur militaire pourvu par l'empereur du ius gladii, le pouvoir de commander des citoyens romains, des légionnaires ${ }^{26}$. On admet généralement que la définition donnée par Dion Cassius correspond aux fonctions du prolégat, même si le mot de $\sigma \tau \rho \alpha \tau \iota \dot{\alpha} \rho \chi \eta \varsigma$, chez Dion Cassius, est assez générique pour désigner des commandements militaires.

On trouve ensuite mention de préfets; trois personnages sont mentionnés: l'anonyme de Fordongianus ${ }^{27}$, l'anonyme de Monti $^{28}$ et L. Aurelius Patroclus, praefectus Sardiniae ${ }^{29}$.

L'anonyme de Fordongianus a le titre de praefectus provinciae [---]. La prudence incite à ne rien développer derrière le terme de provincia, même si l'on pourrait imaginer que comme dans bon nombre de fonctions, un terme précise le ressort administratif. Il s'agit d'une dédicace des civitates barbariae à un empereur dont l'identité a fait l'objet de débats, qui ont abouti à la contestation de la datation du texte: pour A. Taramelli et B.E. Thomasson, il s'agit de l'empereur Auguste. Pour P. Meloni et R. Zucca, il s'agit de l'empereur Tibère. Notre restitution privilégie l'hypothèse de P. Meloni et R. Zucca en

24 Dion Cassius, Histoire romaine, LV, 28, 1, éd. E. Cary, Loeb, Londres, 1960, p. 467. Le terme est d'ailleurs employé au pluriel; Dion Cassius a-t-il voulu évoquer une hiérarchie, ou bien une succession de plusieurs commandants?

25 G. Vrind, De Cassii Dionis vocabulis quae ad ius publicum pertinent, Studia juridica LXII, édition anastatique, Rome, 1971, p. 104-109 l'assimile à un praefectus.

26 J. Šašel, Pro legato, dans Chiron, 1974, p. 467-477.

$27 A E 1921,86=A E$ 1971, 118.

$28 A E$ 1972, 225 et L. Gasperini, Ricerche epigrafiche in Sardegna (I), dans Sardinia antiqua, Cagliari, 1992, p. 292-297.

29 EE VIII, 774, $A E$ 1893, 47, $A E$ 2002, 629 et 630. 
identifiant l'empereur à Tibère sur la foi du titre de préfet porté par le gouverneur de la province. En effet, dans la mesure où nous savons qu'en 14, c'est un prolégat qui a la charge de la Sardaigne, le préfet de la province appartient forcément à une époque ultérieure.

L'anonyme de Monti porte le titre de praefectus provinciae, sans que l'on trouve mention d'une précision quelconque. L'inscription est complète, et la nature de son support, un bloc de roche native émergée d'un torrent, interdit d'envisager une cassure. Le texte mentionne une délimitation, celle des confins du territoire des Balari. Au-delà commence sans doute le territoire d'Olbia. La datation n'est précisée nulle part, mais le titre de préfet de la province incite à dater ce texte des mêmes années que le précédent.

Enfin, L. Aurelius Patroclus porte le titre de praefectus Sardiniae sur quatre milliaires datés de 46 par la titulature de l'empereur Claude, et trouvés à Fordongianus, Busachi, Ruinas et Albagiara.

La différence entre les titulatures saute aux yeux: les deux premiers sont dits praefectus provinciae, le dernier d'entre eux praefectus Sardiniae. On peut à juste titre se demander si cette différence est anodine ou ne l'est pas.

Le premier test est celui du support: il est par exemple difficile d'imaginer que sur une dédicace impériale, on ne respecte pas la titulature officielle. C'est le cas de l'anonyme de Fordongianus (la pierre est malheureusement incomplète) et de L. Aurelius Patroclus. Le cas de l'anonyme de Monti est plus tangent: il s'agit d'un bornage dont la réalisation peut avoir été menée de manière plus fruste.

Le deuxième test est celui de la fréquence de ce terme de provincia dans la titulature d'un préfet. Vérification faite, il n'apparaît que... trois fois. Deux fois en Sardaigne, et une autre fois sur un fragment très mutilé provenant de Vaison-la-Romaine ${ }^{30}$. Tous les autres préfets portent à la suite de leur titre le nom de la province ou du district qu'ils sont censés gouverner, à commencer par le praefectus Aegypti, mais aussi le praefectus Iudaeae, le praefectus Alpium, le praefectus Raetiae, etc.

L'emploi du simple terme de provincia est donc troublant, et peut nous amener à penser que, plus qu'un territoire, c'est une entité juridique que l'on a voulu désigner. Si tel est bien le cas, et tout concours en ce sens, il faudrait en déduire que lorsque sont gravés les deux premiers

30 CIL XII 1355: [pr]aef. pro[---] que la notice de M. Clauss (Deutsche Inschriften online) identifie également comme praefectus pro praetore.

Conimbriga, 49 (2010) 161-181 
textes, les deux îles font partie d'une même entité administrative, d'une même province. On pourrait objecter à cela que le terme de provincia peut manifester une nouvelle création. Mais pourquoi ne pas alors mettre à la suite du terme de provincia le nom du territoire, et ce d'autant plus que ce territoire, s'agissant d'une île, est bien défini? Illustration de cette optique, la restitution habituelle du texte de Fordongianus mentionne un praef. provinciae [Sardiniae]. Mais nous demeurons très réservé à l'égard de cette possibilité, puisque le texte de Monti n'évoque qu'un prefectus provinciae, terme semble-t-il à l'époque suffisant pour désigner le ressort administratif gouverné par ce personnage. Il faut donc en conclure que ces deux textes ont été gravés avant que la séparation des deux îles ne soit effective, puisque l'on continue à évoquer, sur une dédicace comme sur un bornage, le concept de province sans plus définir le ressort territorial concerné. Ce n'est qu'ultérieurement qu'il sera précisé, puisque les milliaires consacrés à Claude désignent explicitement L. Aurelius Patroclus sous le titre de praefectus Sardiniae. Ce fait concrétise en quelque sorte l'adoption d'une dénomination dont plusieurs cas sont bien connus par ailleurs: à titre d'exemple, Ponce Pilate, sur l'inscription de Césarée, est dit praefectus Iudaeae ${ }^{31}$.

A contrario, il est intéressant de noter que certains auteurs anciens pourraient désigner l'ensemble insulaire du simple terme de Sardinia. Ceux-ci définiraient donc par le nom de la plus grande des deux îles l'ensemble de la province. L'épigraphie suit-elle l'exemple de la littérature? En considérant les termes employés dans les titulatures en fonction de leur chronologie, il est aisé d'observer que le terme de provincia est utilisé antérieurement au terme de Sardinia. Ainsi le préfet de la province mentionné sous Tibère apparaît-il avant le préfet de Sardaigne mentionné sous Claude. L'utilisation d'un seul de ces mots pourrait donc démontrer que la conception «épigraphique» du ressort territorial s'exprime en termes plus précis que ceux qu'utilisent les sources littéraires. Ce n'est qu'ultérieurement, sous Vespasien, que les inscriptions montrent une titulature incluant les deux conceptions: Sex. Subrius Dexter est ainsi dit procurateur et préfet de la province de Sardaigne. A partir de ce moment, la titulature du gouverneur inclut les deux termes, et il n'y a plus de confusion possible.

\footnotetext{
$31 \quad A E$ 1963, $104=A E$ 1964, $39=A E$ 1964, $187=A E$ 1971, $477=A E$ 1981, $850=A E$ 1991, $1578=A E$ 1997, $166=A E$ 1999, $1681=A E$ 2002, $1556=A E$ 2005, $1583=A E 2008,1542$.
}

Conimbriga, 49 (2010) 161-181 
Les inscriptions nous livrent en outre les noms de trois préfets qui ont eu en Sardaigne ou en Corse des responsabilités d'un autre type, et qui méritent d'être pris en compte.

Le premier d'entre eux, Sex. Iulius Rufus, est connu par un texte de Préneste: Sex(tus) Iulius S(purii) / f(ilius) Pol(lia) Rufus / euocatus Diui Augusti / praefectus [I] cohortis / Corsorum et ciuitatum / Barbariae in Sardinia ${ }^{32}$. Cette inscription aujourd'hui perdue est datable du règne de Tibère par la mention du Divus Augustus. Cet homme n'est pas originaire de Préneste, ville dont les habitants sont inscrits dans la tribu Menenia, et son origine reste difficile à déterminer. Les rares villes dont les ressortissants soient inscrits dans la tribu Pollia se trouvent non loin de Fanum Fortunae (Fano), sur l'Adriatique, le long de la via Aemilia, entre Faventia (Faïence) au sud et Fidentia (Borgo San Donnino) au nord, aux environs de Turin, dans les cas de Forum Fulvi Valentia (Valenza) et Pollentia (Polenza), et dans le Val d'Aoste, à Eporedia (Ivrea). Or, on ne trouve la trace de Sexti Iulii dans aucune de ces villes, et le gentilice Iulius y est lui-même fort peu diffusé. La tribu Pollia est en revanche la tribu attachée aux camps romains, et $\mathrm{H}$. Devijver, en le rappelant, fait plus que suggérer que cet homme a pu naître dans l'atmosphère des cantonnements des soldats de l'Empire ${ }^{33}$. Sa carrière s'est probablement effectuée à Rome, dans le prétoire, au point d'avoir été distingué par Auguste et rappelé par ses soins après son congé pour accomplir une tâche bien précise.

En effet, avant 14, cet homme a été réintégré dans le service actif par l'empereur Auguste après son temps réglementaire; il a pris la tête d'une cohorte, en l'occurrence la I ${ }^{\text {ère }}$ cohorte des Corses, avec le grade de préfet. Cette charge est habituellement confiée à des centurions du rang, et le fait de voir un évocat nommé à ce poste s'explique par une autre sorte de tâche. Sex. Julius Rufus a aussi, en effet, la responsabilité de la surveillance des cités de la Barbaria (l'actuelle Barbagia), dans

32 CIL XIV 2954 = L.A. Muratori, Novus thesaurus veterum inscriptionum, t. II, Rome, 1754, p. $825=$ Y. Le Bohec, La Sardaigne et l'armée romaine sous le Haut-empire, Sassari, 1990, p. 27-28 et n 5, p. 108-109.

33 H. Devijver, Prosopographia Militiarum Equestrium quae fuerunt ab Augusto ad Gallienum, t. 1, Louvain, 1976, p. 482, n 114 et Id., ibid., t. 5 suppl. 2, Louvain, 1993, p. $2146 \mathrm{n}^{\circ} 114$.

Conimbriga, 49 (2010) 161-181 
le centre de la Sardaigne ${ }^{34}$, et sa charge militaire s'exerce ici sur un territoire: on dispose donc de l'affectation, des moyens et du rayon d'action du personnage.

Ce rayon d'action est limité à un district, et il faut en conclure que Sex. Iulius Rufus est un subordonné du tenant de l'autorité militaire et civile, le gouverneur de l'île. A priori, chronologiquement parlant, il s'agirait plutôt du prolégat que du préfet, même si les exemples de l’Égypte ou de la Maurétanie Tingitane démontrent que les préfets peuvent être ordonnés hiérarchiquement dans une même province. Mais ici, la charge de préfet de cohorte fait de cet homme un subordonné du gouverneur de la Sardaigne, comme le prouvent les diplômes militaires plus tardifs. Le texte est postérieur à la mort d'Auguste, mais cela ne signifie pas que la charge n'a pas été effectuée sous cet empereur, d'autant que c'est Auguste qui le rappelle au service actif. Nous serions assez tenté de voir en Sex. Iulius Rufus un sous-officier du prolégat de Sardaigne, peut-être même de T. Pompeius Proculus, chargé du commandement d'un corps d'armée avec mission de surveillance des cités barbares.

Le deuxième est dit préfet de la cité de Valentia : s'il est difficile de préciser quelle a été sa tâche; le fait qu'il intervienne dans une civitas peut permettre d'imaginer qu'il ait un droit de regard non seulement sur la ville, mais aussi sur son territoire. L'absence de troupes mentionnées dans le texte ne signifie pas qu'il n'en a pas eu sous sa responsabilité; il serait donc le collègue de Sex. Iulius Rufus, chargé de la surveillance du territoire de Valentia ${ }^{35}$.

L'autre préfet mentionné est L. Vibrius Punicus ${ }^{36}$. Son épitaphe, conservée au musée d'Aix-les-Bains, présente une carrière fournie: L(ucio) Vibrio A(uli filio) Vol(tinia) / Punico, praef(ecto) / equitum, / primopilo, trib(uno) / mil(itum), praef(ecto) Corsicae. / $\underline{\text { (aius) }}$ Vibrius Punicus / $\underline{\text { M(arcus) }<\text { Vibrius }>\text { Octauianus / patri merentissimo. }}$. Originaire du territoire de la colonie de Vienne, comme en témoigne

34 Sur le praefectus civitatum, RE XXII, col. 1290-1294 et G. Tibiletti, dans Rend. Ist. Lombardo., Clas. di Lettere, 86 (1953), p. 64 et suivantes. Voir également CIL IX 5363, A. Forci et R. Zucca, M. Arrecinus Helius praefectus civitat(is) [Va]le[n] tinae, dans Epigraphica 69 (2007), p. 209-239 = AE 2007, 692.

35 A. Forci et R. Zucca, ibid. se montrent plus réservés et n'entrent pas dans les attributions du personnage.

36 CIL XII $2455=$ ILN Vienne, 707 avec la bibliographie complémentaire.

Conimbriga, 49 (2010) 161-181 
son appartenance à la tribu Voltinia, L. Vibrius Punicus, à l'issue de sa carrière, est revenu chez lui et y a achevé son existence.

S. Demougin suggère que cette carrière «n'est pas présentée avec rigueur», puisque le commandement d'une aile de cavaliers ne précède pas le primipilat, mais se rencontre habituellement à la suite du tribunat militaire ${ }^{37}$. Elle a donc envisagé de corriger l'ordre des étapes du cursus: L. Vibrius Punicus aurait couronné une carrière de militaire issu du rang comme centurion primipile, et serait successivement devenu tribun des soldats, puis préfet d'une aile de cavaliers, enfin préfet de Corse: il s'agirait alors d'une carrière normale, ou plus justement parmi les plus fréquemment recensées avant la réforme des milices équestres par Claude ${ }^{38}$.

Au contraire, B. Rémy (ILN Vienne, 707) recense dans l'ouvrage de S. Demougin d'autres exemples atypiques: il suggère que l'ordre annoncé n'est pas dû au hasard dans la mesure où les deux personnages concernés ont exercé avant le primipilat qui une préfecture de cohorte ${ }^{39}$, qui une préfecture d'un autre type ${ }^{40}$. Cependant, il faut se souvenir que la préfecture d'une cohorte peut être donnée à des centurions ${ }^{41}$, que ces deux carrières reflètent donc un avancement normal, et qu'elles ne sont par conséquent pas comparables à celle de L. Vibrius Punicus.

Un autre chevalier du nom de L. Aponius suit en revanche une carrière que l'on pourrait envisager de comparer avec celle du préfet de Corse: il fut en effet successivement préfet des cavaliers, tribun militaire

37 S. Demougin, Prosopographie des chevaliers romains julio-claudiens, Rome, 1992, no 398, p. 328-329 [= Prosopographie].

38 H. Devijver, Suétone, Claude 25, et les milices équestres, dans Ancient Society 1 (1970) p. 68-81: Claude réforme le service militaire et, si sa réforme ne connaît pas de lendemain, ou sous une autre forme, elle empêche surtout les anciens primipiles d'accéder aux grades équestres. Cf. H.-G. Pflaum (Les procurateurs équestres sous le Haut Empire romain, Paris, 1950, p. 215) qui évoque la fusion des fonctions équestres en une hiérarchie sous Claude: «le titre de prolégat disparaît et le titre de praefectus prend une autre signification. Il s'applique alors à des administrateurs subordonnés gouvernant à l'intérieur des provinces des territoires limités. Les anciens praefecti des districts militaires sont assimilés aux procurateurs de Judée et de Cappadoce».

39 S. Demougin, Prosopographie, $\mathrm{n}^{\circ}$ 252, p. 218-219.

40 S. Demougin (Prosopographie, ${ }^{\circ} 410$, p. 334-335) suggère qu'il s'agit de la praefectura navium au mouillage à Fréjus ; mais il peut s'agir d'une autre préfecture, plus banalement d'une préfecture de cohorte.

41 S. Demougin, Ordre équestre, p. 346.

Conimbriga, 49 (2010) 161-181 
et préfet de camp ${ }^{42}$. Les carrières connaîtraient une similitude encore plus grande si l'on admettait d'introduire entre la préfecture d'aile et le tribunat légionnaire la mention du primipilat; mais les restitutions privilégient, de manière peut-être contestable, la restitution du primipilat avant la préfecture d'aile.

Il n'en reste pas moins que l'un des éléments de ces carrières prête à polémique: en effet, dans ces deux cas, la praefectura equitum se trouve à une place inhabituelle; ce commandement allait en effet d'habitude à d'anciens tribuns, ce que n'étaient alors ni L. Vibrius Punicus, ni L. Aponius. La clé de cette énigme ne se trouverait que dans des circonstances exceptionnelles, le remplacement d'un officier manquant ou la levée d'une troupe par un particulier, solutions au demeurant peu satisfaisantes, car elles ne rencontrent que peu de confronts. Mais une autre hypothèse pourrait être envisagée: peut-être, plus que d'une erreur de positionnement, pourrait-il tout simplement s'agir d'une confusion provoquée par des dédicants certes désireux d'honorer la mémoire de leurs parents, mais peu au fait des hiérarchies et des terminologies militaires: L. Vibrius Punicus et L. Aponius auraient ainsi tout simplement pu être préfets de cohortes montées, assimilées par les dédicants à des préfectures de cavaliers. Leur cursus rentrerait ainsi dans la normalité des carrières pré-claudiennes.

Dans tous les cas, l'ensemble des éléments de la carrière de L. Vibrius Punicus permettent de la situer avant la réforme claudienne, et l'importance de cette carrière, marquée par le passage dans l'ordre équestre, suggère que cet homme n'a rien d'un subordonné. Les rares auteurs qui ont écrit à propos de cet individu problématique n'ont pas pris position, parfois se sont contredits ${ }^{43}$. Mais il faut noter que L. Vibrius Punicus, au contraire de nombreux autres individus de position subalterne (à commencer par Sex. Iulius Rufus), n'est pas défini comme étant praefectus in Corsica; ceci pourrait nous faire admettre qu'il s'agit bien ici du premier gouverneur autonome connu de la Corse. L. Vibrius Punicus pourrait donc au mieux être contemporain de L. Aurelius Patroclus mais, à considérer la paléographie de son épitaphe, il est certainement plus ancien.

42 S. Demougin, Prosopographie, ${ }^{\circ}$ 202, p. 177-178.

43 S. Demougin, Ordre équestre, p. 376 répertorie L. Vibrius Punicus parmi les «officiers équestres promus directement, après le tribunat militaire ou la préfecture d'aile, au poste de primipile».

Conimbriga, 49 (2010) 161-181 
La datation de son mandat est donc incertaine, mais nous pouvons, à l'aide des éléments que nous avons définis, en tenter une approche: si l'on admet qu'à la suite du prolégat appelé à d'autres tâches, des préfets de la province ont assuré l'administration des deux îles, il faut placer le mandat de L. Vibrius Punicus après les débuts du règne de Tibère, auquel est consacrée, sous les auspices du préfet de la province, la dédicace des cités de la Barbaria; il est alors soit le «lieutenant» du préfet de la province lors du mandat de celui-ci (comme Sex. Iulius Rufus), soit préfet à part entière de la Corse après la division des administrations des deux îles. Comme son cursus est antérieur à la réforme des milices équestres, ces éléments nous permettent de restreindre à un laps de temps de 30 ans au plus, entre les années 15 et 45, l'époque où L. Vibrius Punicus a pu être en charge de la Corse.

Ceci nous amène naturellement à mettre en parallèle les divers titres de ceux qui ont dirigé les unités armées et l'administration des îles. Les titres complexes sont à mettre en relation avec le titre plus simple de préfet de la province, attesté à Fordongianus (avec une lacune) et à Monti, qui prouve l'existence d'un seul préfet pour l'ensemble du territoire de la province. Il reste à savoir si les autres préfets ont eu un commandement indépendant ou s'il ont été placés sous l'autorité du gouverneur de la Sardaigne. Dans ce dernier cas, leur commandement de type militaire devait tout de même inclure une certaine capacité d'initiative.

Par ailleurs, ces préfets ont-ils exercé leur autorité de manière contemporaine, ou doit-on comprendre que leurs tâches ont été exercées à des époques différentes? Le titre de préfet des cités barbares est également à mettre en relation avec le titre de préfet de Corse. Les deux préfets n'auraient-ils pas été des subordonnés du prolégat? Donner une réponse est difficile.

Notons cependant qu'en 46, le gouverneur de la Sardaigne auquel Claude a confié l'entretien des routes se dit préfet de Sardaigne, et non pas préfet de la province, tout comme L. Vibrius Punicus est dit préfet de Corse. La conception selon laquelle la Corse et la Sardaigne ne forment qu'une seule province ne dure donc vraisemblablement pas au-delà du règne de Tibère. Considérées comme des districts militaires, les îles sont probablement gérées séparément par les commandants des unités qui y maintiennent l'ordre avant que la séparation effective ne soit entérinée par Claude qui y installe deux procurateurs. 
Il faut à présent comprendre pourquoi l'administration de la province de Corse et Sardaigne a subi de telles modifications dans un espace de temps aussi court. Nous n'avons pas d'indication sur les motifs qui ont pu pousser le préfet unique a être remplacé par un préfet de Corse et un préfet de Sardaigne. Peut-être s'agissait-il simplement de raisons techniques. Mais ceci est probablement autant dû à des circonstances extérieures qu'à des problèmes internes, insoumission et brigandage, tous deux endémiques.

\section{Les raisons de l'évolution d'un statut}

La province, constituée comme telle au III ${ }^{\mathrm{e}}$ siècle av. J.-C., revient au Sénat lors du partage de 27 av. J.-C. Elle est alors confiée à des proconsuls «itinérants», si l'on admet qu'ils devaient faire la navette entre la capitale régionale, Karales (Cagliari) et la capitale de la Corse, Aleria. Le gouverneur C. Mucius Scaevola a ainsi par exemple laissé les traces de ses évergésies dans les deux villes.

C'est en 6 de notre ère que se situe une rupture: Dion Cassius mentionne l'envoi de $\sigma \tau \rho \alpha \tau \iota \dot{\alpha} \rho \chi \varepsilon l \varsigma$, de gouverneurs militaires équestres aux compétences étendues, qu'un texte désigne sous le terme de pro legato en mentionnant l'un de ceux-ci, T. Pompeius Proculus (13/14). Y. Le Bohec ajoute à cette constatation une observation intéressante: selon lui, la présence de ces commandants militaires pourrait avoir été discontinue entre 6 et $14^{44}$. Cette proposition se heurte pourtant à Dion Cassius, qui n'évoque jamais le retour de l'île à une administration sénatoriale, et ne mentionne même jamais que les troupes ont été retirées à un moment quelconque. Par ailleurs, Tacite mentionne qu'en 19, 4000 affranchis sont envoyés en Sardaigne pour combattre le brigandage ${ }^{45}$.

44 Dans le même ordre d'idée, B.E. Thomasson (Zur Verwaltungsgeschichte der Provinz Sardinia, dans Eranos LXX (1972), p. 72-81) pense que le gouverneur de la province de Corse-Sardaigne a fort bien pu voir fluctuer son titre entre 6 et 14 , passant de prolégat à préfet, puis redevenant un prolégat. Cette proposition se base sur la datation de l'inscription de Fordongianus mentionnant les cités de la Barbaria, qu'il attribue à l'époque d'Auguste. Sans souscrire à cette suggestion, que notre datation de l'inscription infirme en grande partie, nous ne pouvons totalement écarter cette possibilité.

45 En 19, on envoie 4000 hommes «de la classe des affranchis (libertini est le terme employé par Tacite), infectés de ces erreurs (les superstitions égyptiennes et 
Soit le phénomène est endémique, soit il se manifeste de manière régulière, sinon permanente, et cela explique la nécessité de corps de troupes sur place. Cette politique est couronnée de succès, puisque nous voyons le préfet de la province poser le bornage de Monti entre l'ager d'Olbia et les territoires de la peuplade des Balari et que les cités de la Barbaria pacifiée lui rendent hommage à travers l'empereur; le statu quo est donc maintenu.

Mais si le brigandage ou la piraterie impliquent l'envoi de troupes, ils n'expliquent pas un envoi continu et renouvelé, d'abord en 6, ensuite en 19, puis leur maintien sous la forme de cohortes d'auxiliaires. Si le brigandage est endémique, il faut que les troupes de 6 soient parties, et qu'elles aient été remplacées par ces affranchis.

Un événement peut s'avérer être à la source de ces variations: il se déroule en Afrique, sous l'impulsion d'un indigène. Il s'agit de la révolte de Tacfarinas, qui a lieu en diverses phases de 17 à 24, a pour théâtre d'opérations le sud de l'Afrique Proconsulaire et de la Maurétanie et se déroule schématiquement en plusieurs étapes.

En 17, le maure Tacfarinas dresse les poulations africaines spoliées de leurs terres contre les colons et organise la guérilla contre les troupes romaines. En 17-18, le proconsul Furius Camillus, avec la III légion Auguste, les corps auxiliaires et les troupes de Juba II arrive à contenir la révolte. En 18-21, le proconsul L. Apronius, qui dispose des mêmes forces, subit plusieurs cuisantes défaites avant de reprendre le dessus à Thala, où Tacfarinas est battu. En 21-23, Q. Iunius Blaesus dispose des mêmes troupes et, en renfort, de la IX ${ }^{\mathrm{e}}$ légion Hispana, venue de Pannonie en $22^{46}$. Il poursuit la contre-guérilla avec des troupes réparties en petits effectifs, remporte la victoire qui lui vaut les ornements triomphaux et le titre d'imperator. La IX ${ }^{\mathrm{e}}$ légion Hispana est rappelée

judaïques), en âge de porter les armes» pour réprimer le brigandage (Tacite, Annales, II, LXXXV; Flavius Josèphe, $A$. J. XVIII, 3, 5, 84; Suétone, Tibère 36). Il ne s'agit pas de légionnaires, car le nombre de 4000 hommes est inférieur à l'effectif d'une légion, et pas davantage d'auxiliaires, puisque le chiffre excède de beaucoup celui des effectifs affectés à ces corps de troupe. Il pourrait correspondre à celui d'une colonie de peuplement, avec femmes et enfants. Il s'agirait alors non d'une opération ponctuelle de pacification, mais de l'installation d'une colonie affectée au maintien de l'ordre, cf. G. Marasco, Tiberio e l'esilio degli Ebrei in Sardegna nel 19 d.C., dans Afr.Rom. VIII (1990), p. 649-659.

46 M. Silvestrini, Africa proconsolare: note epigrafiche, dans Afr.Rom. III (1985), p. 245, n. 15 et V.A. Sirago, Tacfarinas, dans Afr.Rom. V (1987), p. 200.

Conimbriga, 49 (2010) 161-181 
en Pannonie ${ }^{47}$. Enfin, en 23-24, P. Cornelius Dolabella ne dispose plus que des troupes normalement affectées à la province. Tacfarinas assiège Thubursicu Numidarum, puis est chassé et battu avant de se suicider.

En suivant le déroulement de cette révolte, on pourrait observer une continuité, sinon plusieurs. Il s'agit d'une guérilla menée sur un vaste théâtre d'opérations. Ce type de conflit nécessite des troupes en nombre afin de quadriller le terrain.

Durant la première phase (17-18), les troupes ordinaires suffisent pour contrer la révolte et le proconsul obtient les ornements triomphaux. Dans un deuxième temps (18-21), avec les mêmes troupes, les Romains sont battus, puis réussissent à remonter la pente. Ce n'est que dans un troisième temps (21-23) que l'on décide d'envoyer en Afrique une légion de plus, la IX ${ }^{\mathrm{e}}$ Hispana. Tacfarinas est battu et Q. Iunius Blaesus obtient les ornements triomphaux. Enfin, dans une quatrième phase (23-24), la IX ${ }^{\mathrm{e}}$ légion Hispana retourne en Pannonnie et P. Cornelius Dolabella bat avec difficultés Tacfarinas.

Somme toute, la présence de la IX ${ }^{\mathrm{e}}$ légion était indispensable $\mathrm{e}^{48}$. La décision de la transférer en Afrique n'a pas été prise à la légère, et il est probable que c'est la succession des revers militaires qui a motivé cet acte. Une question se pose alors: le besoin de troupes étant permanent, pourquoi n'avoir pas fait mouvoir les unités de Sardaigne avant de faire venir une légion de Pannonie? D'une part, il est beaucoup plus rationnel d'imaginer une augmentation progressive des forces: on se contente dans un premier temps des troupes ordinairement cantonnées dans la province, on fait appel dans un deuxième temps à des renforts auxiliaires (ou cantonnés non loin), enfin on appelle à la rescousse les renforts légionnaires. D'autre part, la Sardaigne est à l'époque la province pourvue de troupes la plus proche de l'Afrique. Il est donc fort possible que dans un deuxième temps, c'est de là qu'on ait fait venir des troupes composées de légionnaires ou d'auxiliaires ${ }^{49}$. Du

47 Tacite, Annales, IV, 5 mentionne cependant en 23 deux légions en Afrique et deux légions en Pannonie.

48 Il existe une abondante littérature à propos des motifs qu'a eus Tibère de la retirer en 23, entre autres le fait qu'il fallait ménager Q. Iunius Blaesus, l'oncle de Séjan, et qu'il était par ailleurs nécessaire de ne pas laisser trop longtemps dégarni le front du Danube.

49 A. Gonzalez (La révolte comme acte de brigandage. Tacite et la révolte de Tacfarinas, dans Afr.Rom. XII (1996), p. 948) suggère que le bellum Gaetulicum s'achève en 6. Les troupes ainsi libérées pouvaient alors aller en Sardaigne. Il est dans 
reste, la preuve de la mobilité des troupes sardes est matérialisée par une découverte effectuée à Mila (Milev): il s'agit de l'épitaphe d'un soldat sarde : Optatus / Sadecis / f(ilius) decurio / co(ho)rti(s) Lusita/ na v(ixit) a (nnis) $L V / \operatorname{Sardus}^{50}$. Optatus, fils de Sadex, d'origine sarde, décurion d'une cohors Lusitana, est mort à l'âge de 55 ans. Ce texte est daté par Y. Le Bohec du milieu du $\mathrm{I}^{\mathrm{er}}$ siècle selon le formulaire employé, l'onomastique du personnage et son recrutement. Ce Sarde, pourvu d'un grade non négligeable dans une cohorte montée d'auxiliaires, est donc mort en Afrique, sans que nous puissions déterminer s'il était en service ou à la retraite. Si nous admettons qu'il avait, eu égard à son âge, achevé ses 25 ans réglementaires, peut-être s'est-il engagé dans les années 20, à l'époque des événements cités.

Cet indice ne serait qu'anecdotique, si nous ne savions que la cohors Lusitanorum était en garnison en Sardaigne au I ${ }^{\text {er }}$ siècle: elle y est en effet connue par un texte d'Austis mentionnant un tubicen $^{51}$. Comme il est sûr qu'elle se trouvait en garnison en Numidie durant le troisième quart du $\mathrm{I}^{\mathrm{er}}$ siècle, une chronologie pourrait être définie ainsi: la cohorte de Lusitaniens cantonnée à Austis dès le début du $\mathrm{I}^{\mathrm{er}}$ siècle est transférée en Afrique à l'occasion de la révolte de Tacfarinas ; elle $\mathrm{y}$ reste définitivement et Optatus meurt à Milev plusieurs années après.

Il semble également fort possible que l'on ait transféré en Afrique des unités légionnaires cantonnées en Sardaigne, et c'est probablement en fonction de cette «déqualification» des troupes restées dans l'île que le gouverneur de la province de Corse et Sardaigne n'a plus été un prolégat, mais un préfet chargé des îles et disposant éventuellement de subalternes chargés de secteurs spécifiques, à égalité de titre, sinon de fonction; l'un de ceux-ci a eu la charge de la Corse, et ce prédécesseur de L. Vibrius Punicus a pu avoir une liberté de décisions suffisante pour être finalement autonome. La voie était ouverte vers la modification statutaire qui, aux alentours de 46, était effective.

ce cas possible qu'au début de la révolte de Tacfarinas, en 17, le légat Furius Camillus ait pu ordonner ce qui était un simple rapatriement de contingents.

50 AE 1929, 169, Mila (Milev).

51 CIL X, 7884, Augustis (Austis).

Conimbriga, 49 (2010) 161-181 


\section{Conclusion}

Pour tirer un bilan de cette étude effectuée sur la base de sources directes, nous pensons que la variation et la succession des titres des gouverneurs équestres de la province de Corse et Sardaigne, prolégat, préfet de la province, puis préfet de Sardaigne ou préfet de Corse, permettent d'effectuer une distinction entre des titres en exacte conformité avec ceux qui sont recensés dans le reste du monde romain et des appellations tout à fait inédites.

L'existence de ces nouveaux titres ne doit rien au hasard; elle revêt une signification. C'est en fonction des compétences et des responsabilités militaires et territoriales de ces gouverneurs que l'administration des îles a été étape par étape transformée entre le règne de Tibère et le règne de Claude, jusqu'à aboutir à la constitution de deux entités séparées ${ }^{52}$ : nous savons que cette division est un fait avant 69 , date à laquelle est mentionné sur la table d'Esterzili le procurateur impérial M. Iuventius Rixa, l'un des prédécesseurs du proconsul Helvius Agrippa, alors que Tacite évoque cette même année le procurateur de Corse Decimus Picarius, et nous pouvons même préciser qu'elle est probablement déjà acquise lorsque les milliaires sardes mentionnent un praefectus Sardiniae en 46 de notre ère.

Cette évolution est plus vraisemblablement due à des circonstances extérieures, la révolte africaine de Tacfarinas, déclenchée en 17, qu'à des causes directement liées à la population rebelle ou à la géographie capricieuse des deux îles. Le passage d'une administration à une autre est donc au fond plus un fait casuel, une adaptation aux circonstances, qu'une pensée mûrement réfléchie, et il est assez décourageant de constater qu'il n'y a de politique romaine vers les îles qu'en terme de pragmatisme. Il n'en reste pas moins que d'adaptation en adaptation, l'Empire a approuvé la constitution en province de chacune des deux îles.

Si les premiers fonctionnaires équestres «indépendants» peuvent donc probablement être recensés sous le règne de Tibère, c'est plus sûrement au début du règne de Claude qu'a été entérinée une situation acquise dans les faits depuis près de vingt ans, la séparation des administrations insulaires, par l'érection d'Aleria en capitale de la nouvelle province de Corse.

52 Comme l'avait déjà suggéré P. Meloni, La provincia romana di Sardegna, dans $A N R W, 1988$, p. 467.

Conimbriga, 49 (2010) 161-181 